\title{
Nutritional Garden: A Nutrition Sensitive Promising Approach towards Improving Nutritional Security in Farm Households
}

\author{
Manisha Choudhary ${ }^{1}$, S. P. Singh ${ }^{1 *}$ and Chanchala Rani Patel ${ }^{2}$ \\ ${ }^{1}$ Krishi Vigyan Kendra, Raigarh, (C.G.), India \\ ${ }^{2}$ Indira Gandhi Krishi Viswavidyalaya, Raipur (C.G.), India \\ *Corresponding author
}

\section{Keywords}

Nutritional garden, Nutritional security, Farm households

\section{Article Info}

Accepted:

10 January 2021

Available Online:

10 February 2021
The present study was carried out on nutritional garden towards improving nutritional security in farm households. India is one of the leading country in vegetable production but malnutrition problem especially caused by micronutrient deficiencies are in general family diet. The underlying cause may be lack awareness, illiteracy, low affordability, inadequate availability of vegetables and fruits. Recommended dietary allowances (RDA), daily intake of vegetables should be $300 \mathrm{gm} /$ person including roots and tubers, green leafy and other vegetables. Malnutrition is a serious nutritional problem, resulting in different types of diseases, hampering physical growth and retarding brain development. Nutritional garden practices with strict adherence to sustainable technologies will be an everlasting solution. It ensures balanced household consumption of vegetables and fruits in their daily food habits and prevents nutritional maladies. The underutilized backyards of landless women not only serve the entire family with nutritious food but also get alternate livelihood. Homestead production of vegetables and fruits help combat malnutrition and diversify diets. Improved nutritional garden is better than traditional homestead garden. The improved nutritional garden involves many crops that can be repeatedly harvested to meet a family's vegetable needs and economic return. Hence, nutritional garden is an important strategy to improve household nutritional security. The result of the study showed that nutritional garden has proved a feasible livelihood strategy for resource poor farm households and improvement in availability of vegetables for consumption in terms of nutrient as well as calorie intake and economic performances

\section{Introduction}

Nutritional garden are in culture since ancient times and are growing vegetables, fruits, and other food crops in underutilized backyards of landless farm households not only serve the entire family with nutritious food but also get alternate livelihood. Production of vegetables in the world was almost 1010 million tons (FAO, 2011). Asia produces and consumes more than $70 \%$ of the world's vegetables. China has always been a large contributor and currently produces over $50 \%$ of the world's vegetables. India is the second largest producer of vegetables in the world but at almost a six-fold lower level than China. 
Worldwide the area of arable land devoted to vegetables is expanding at $2.8 \%$ annually, higher than fruits $(1.75 \%)$, oil crops $(1.47 \%)$, root crops $(0.44 \%)$ and pulses $(0.39 \%)$ and at the expense of cereals $(-0.45 \%)$ and fibre crops (-1.82\%) (FAO, 2009). Leafy vegetables - of which the leaves or young leafy shoots are consumed-were the most often utilized ( $53 \%$ of the total), followed by fruit vegetables $(15 \%)$, while vegetables with belowground edible organs comprised $17 \%$. Many vegetable crops have more than one part used (Dias and Ortiz, 2014). Homestead production of vegetables, fruits, spices, medicinal and aromatic plants help combat malnutrition, diversify diets and are boon to mankind in several ways. Vegetables are major source of vitamins, minerals, and fibers; their nutritive and medicinal values in human beings. Indian Council of Medical Research (ICMR) recommendations every individual should consume at least $300 \mathrm{~g}$ vegetables and $100 \mathrm{~g}$ fresh fruits /day (green leafy vegetables $-50 \mathrm{~g}$, other vegetables 200 $\mathrm{g}$, roots and tubers $-50 \mathrm{~g}$ ). Pregnant women should consume $100 \mathrm{~g}$ leaf vegetables/day. Several nutritional problems of human beings are protein energy malnutrition (PEM), micro nutrient deficiencies like vitamin A deficiency (VAD), Iron deficiency anaemia (IDA), Iodine deficiency disorder (IDD) and Vitamin B complex deficiencies (NIN, 2011). Nutritional garden can be a profitable proposition in India which is predominantly vegetarian and, as such, a large number of nutrients are obtained from vegetables for a balanced diet. Due to inadequate consumption of vegetables, deficiency of micro-nutrients especially of iron, vitamin A and iodine are prevalent in the developing world (Hall et al., 2009; Kanungsukkasem et al., 2009; Satheannoppakao et al., 2009; Leenders et al., 2013; NCCDPHP, 2013). National institute of nutrition (NIN) recommended for these problems are: (i) eat variety of foods to ensure balanced diet, (ii) eat protective foods rich in vitamins and minerals. (ii) eat folate rich foods for haemoglobin synthesis. (iv) eat plenty of fruits and vegetables which are rich sources of micro and macronutrients. Vegetables are rich sources of nutritional bioactive compounds. They are important sources of protective nutrients like vitamins, minerals, antioxidants, folic acid and dietary fibres. The diversified and highly nutritive vegetables are affordable and cost effective solution to hidden hunger and malnutrition. India has a rich heritage of indigenous fruits and vegetables. They are not only rich in minerals and vitamins but also, contribute in a big way in maintaining health, overcoming hunger and malnutrition. Per capita on average needs a daily diet which should provide 2800 calories, $55 \mathrm{~g}$ protein, $450 \mathrm{mg}$ $\mathrm{Ca}, 20 \mathrm{mg}$ Fe, $3000 \mathrm{mg} \beta$ - carotene, $50 \mathrm{mg}$ vitamin $\mathrm{C}, 100 \mathrm{mg}$ folic acid, $1.0 \mathrm{mg}$ vitamin $\mathrm{B}, 1.4 \mathrm{mg}$ thiamine, $1.5 \mathrm{mg}$ riboflavin, $19 \mathrm{mg}$ niacin and $5 \mathrm{mg}$ vitamin $\mathrm{D}$. Vegetables are good source of all these nutrients (Sharma, 2009). Vegetables help combat malnutrition and diversify diets. Dietary diversification balances the diet by enhancing the supply of essential micro-nutrients leading to improved health, such as improving functions of the whole body, disease prevention, and delayed disease progression (Somsri et al., 2016), enhanced thinking ability and increased efficiency (Jindal and Dhaliwal, 2017). Examples include improvement in microvascular reactivity (Macready et al., 2014), better cognitive performance (Nyaradi et al., 2014), decreased risk of colorectal cancer (Zhong et al., 2014), reduce the risk of overweight (Howarth et al., 2001; Tohill, 2005), coronary heart disease (Dauchet et al., 2006; He et al., 2007; Hartley et al., 2013), and reduced risk of kidney disease (Goraya $e t$ al., 2013). Melina (2012) suggested that "a menu filled with seasonal fruits and vegetables could provide a big nutritional boost", and vegetables were packed with fiber and water, and were low in fat, they decreased 
the calorie density of diet, while boosting overall nutrition. It was evident from the literature that home gardens are a part of agriculture and food production systems in many developing countries and are widely used as a remedy to alleviate hunger and malnutrition in the face of a global food crisis (Johnson et al., 2000). Mitchell and Hanstad (2004) reported that home garden provided multiple social benefits such as enhancing food and nutritional security, empowering women, promoting social justice and equity, and preserving indigenous knowledge and culture and so on. One of the primary objectives of this study is to develop nutrition garden model especially for urban and periurban households which ensure healthy diet that contains adequate quantities of vitamins and macro and micro-nutrients by producing diverse kinds of vegetables. Malnutrition among the pre- school children can be used to determine the need for nutritional surveillance, nutritional care or appropriate nutritional intervention program in community. National Family Health Survey-4 (2015-16) data revealed 42.3\% (Chhattisgarh$39.6 \%$ ) of pre- school children are stunted and $53.1 \%$ (Chhattisgarh -39.2\%) are under weight due to chronic under nutrition. India accounts for more than 3 out of every 10 stunted children in the world. The intake of protective foods like pulses, vegetables, milk and fruits are very low which leads to many nutritional deficiency disorders. Malnutrition in Indian children continues to be of higher level with $42.3 \%$ being under weight, $58.8 \%$ stunted and $11 \%$ wasted (Indumathi et al., 2012). In India malnutrition and poor health status is a common problem of rural areas. It retards growth, increases the risk and duration of illness, reduces work output and slows social and mental development. For poor households, vegetables and fruits are often the only sources of micronutrients in the family diet. Homestead production of fruits and vegetables provides the households with direct access to important nutrients that may not be readily available or within their economic rich. So, home gardening would be a good means to improve household food security (Talukder et al., 2002). Therefore, more attention towards home gardening as a strategy to enhance household food security and nutrition is to be needed. Globally, nutrition gardening contributes to household food security by providing direct access to food that can be harvested, prepared and fed to family members, often on a daily basis. Even very poor, landless or near landless people practice gardening on small patches of homestead land, vacant lots, roadsides or edges of a field, simple hydroponics, or in containers. Gardening may be done with virtually no economic resources, using locally available planting materials, green manures, "live" fencing and indigenous methods of pest control. Thus, home gardening at some level is a production system that the poor can easily enter. Gardening provides a diversity of fresh foods that improve the quantity and quality of nutrients available to the family (Marsh, 1998). Nutrition gardening is especially important in rural areas where people have limited income-earning opportunities and poor access to markets. These gardens are also becoming an increasingly important source of food and income for poor households in peri-urban and urban areas (Christanty, 1990 and Shackleton et al., 2008). The major vegetable crops of Chhattisgarh are tomato, brinjal, potato, okra, onion, garlic, ginger, coriander, chilli, cauliflower, cabbage, radish, beet root, beans, spounge guard, bitter guard, green leafy (bhaji) and cucurbits etc. Vegetables are very important part of a good diet as they contain various nutrients for many body functions. These vegetables also provide taste, palatability, better digestibility to us and increase the appetite. Vegetables are suitably grown in backyard space as they are mostly seasonal crops. The nutritional garden is 
generally located close to the house and is used for growing vegetables, fruits, and other food crops (Jana, 2015). It not only saves our money and time but also can provide a healthy, useful and environment friendly. Home gardens can help us in recycling of household waste especially when a compost pit is developed. One of the easiest ways of ensuring access to a healthy diet that contains adequate macro- and micronutrients is to produce many kinds of foods in the home garden. The challenge of increasing vegetables consumption is a major concern for health professionals. An estimated 6.7 million deaths worldwide were attributed to inadequate fruit and vegetable consumption in 2010 (Lim et al., 2012). Further, the vegetables reaching the market contain high amount of pesticide residues, it is of special interest to the consumers to grow their own vegetables for domestic consumption. Application of pesticides for insect-pest and disease management is discouraged in the nutrition gardens. Therefore, present study was conducted on nutritional garden towards improving nutritional security in farm households.

\section{Materials and Methods}

The study was carried out on nutritional garden towards improving nutritional security in farm households to developed the nutritional garden during 2017-18, 2018-19 and 2019-20. Nutritional garden developed in aganwadi centers, primary schools and tribal boys and girls hostels of nutrismart and adopted villages- Bhasgadhi, block- Tamnar, Koriyadadar and Loing, block- Raigarh,. and Hamirpur, block- Tamnar district-Raigarh (C.G.). The awareness programmes (trainings) was organized for upgrade the knowledge of farm households, boys and girls regarding the importance of the nutritional garden and the technical aspects of its establishment. Farm households, boys and girls were selected through convenient sampling technique based on their willingness and interest to establish nutritional garden in their land or backyard space to ensure nutrition security. It has also been ensured that the family size should be of 4-6 members. For individual farm households, an area of $250 \mathrm{~m}^{2}$ was taken for the establishment of nutritional garden. During the study Krishi Vigyan Kendra, Raigarh (C.G.) was provided the quality seed and planting material of improved varieties in kharif, rabi and zaid seasons. For the establishment of nutritional garden quality planting mterials and seeds of papaya, durumstick, lemon and cury leaves and seven type of leafy vegetable (spinach, radish, amaranthus, fenugreek, chickpea, bathuva, musturd etc.), pumkin, bottal guard, bitter guard, sponge gourd, cow pea, green pea, tomato, brinjal, okra, chilli, coriander, carrot, beet root, cauliflower, cabbage, were provided among the beneficiaries. To assess the impact nutritional garden and average yield per unit was obtained. The dietary survey and feedback information from the selected households in order to assess their food consumption pattern pre and post was also recorded. The nutrient availability to every individual of farm households as per food composition. Nutrient availability was compared with the recommended dietary allowances. Per capita on average needs per day diet which should provide 2800 calories, $55 \mathrm{~g}$ protein, $450 \mathrm{mg} \mathrm{Ca}, 20 \mathrm{mg} \mathrm{Fe}, 3000 \mathrm{mg}$ $\beta$ - carotene, $50 \mathrm{mg}$ vitamin $\mathrm{C}, 100 \mathrm{mg}$ folic acid, $1.0 \mathrm{mg}$ vitamin $\mathrm{B}, 1.4 \mathrm{mg}$ thiamine, 1.5 $\mathrm{mg}$ riboflavin, $19 \mathrm{mg}$ niacin and $5 \mathrm{mg}$ vitamin D. Vegetables are good source of all these nutrients (Tables 1 and 2) (Sharma, 2009). "Eating a rainbow" of vegetables means including as much coloured vegetables into our daily diet. As the colours are an indication of vitamins and other nutrients, the coloured vegetables enhance the body's ability to prevent and fight diseases. The multitude of phytochemicals present in vegetables acts as 
anti-oxidant, anti-allergic, anti-carcinogenic, anti-inflammatory, anti-viral and antiproliferative. Pigments from paprika are used as natural colourants in medicines and food items. Some of the nutraceutically rich vegetable crops are red and black carrot, beet root, tomato, chilli and broccoli. Based on colour vegetables are grouped (Table 4), (Singh, 2012).

\section{Results and Discussion}

The study was carried out on nutritional garden towards improving nutritional security in farm households to develop the nutritional garden with scientific interventions. Capacity building programmes (trainings) of farm households helps in creating awareness, increasing the knowledge about innovative technologies and empowerment. During awareness programmes on nutritional garden several constraints raised by farm households have also been resolved. The results found that the vegetables harvested were utilized for household consumption and the excess vegetables were distributed among the neighboring farm households. Per capita availability of nutrients/day increased after establishment of nutritional garden intervention (Awasthi et al., 2016). It is obvious from that $3.93 \%$ protein, $30.35 \%$ iron, $32.61 \%$ calcium, $36.32 \%$ beta-carotene, $144 \%$ vitamin $\mathrm{C}$ and $15.49 \%$ folic acid of RDA were more available to individual (Singh et al., 2018). Establishment of nutritional garden as a community level strategic intervention programme in 50 farm households revealed that the percent intake of Calcium, Iron, Vitamin A and Vitamin $\mathrm{C}$ at the end of the intervention showed an increase of $89 \%, 61 \%, 83 \%$ and $65 \%$ respectively (Vani and Usha, 2013 and Chayal et al., 2013) (Tables 1-5).

Table.1 Nutritional composition in vegetables per $100 \mathrm{~g}$ edible portion

\begin{tabular}{|l|c|c|c|c|c|c|}
\hline \multicolumn{1}{|c|}{ Vegetable crops } & $\begin{array}{c}\text { Energy } \\
\text { (Kcal) }\end{array}$ & $\begin{array}{c}\text { Moisture } \\
(\mathbf{g})\end{array}$ & $\begin{array}{c}\text { Protein } \\
(\mathbf{g})\end{array}$ & $\begin{array}{c}\text { Fat } \\
(\mathbf{g})\end{array}$ & $\begin{array}{c}\text { Carbo } \\
\text { hydrates }(\mathbf{g})\end{array}$ & $\begin{array}{c}\text { Fibre } \\
(\mathbf{g})\end{array}$ \\
\hline Amaranth & 45.0 & 85.7 & 4.0 & 0.5 & 6.1 & 1.0 \\
\hline Asparagus & 26.0 & 91.7 & 2.5 & 0.2 & 5.0 & 0.7 \\
\hline Basella & 32.0 & 90.8 & 2.8 & 0.4 & 4.2 & - \\
\hline Bittergourd & 25.0 & 92.4 & 1.6 & 0.2 & 4.2 & 1.7 \\
\hline Bottle gourd & 12.0 & 96.1 & 0.2 & 0.1 & 2.5 & 1.5 \\
\hline Brinjal & 24.0 & 92.7 & 1.4 & 0.3 & 4.0 & - \\
\hline Broad bean & 48.0 & 85.4 & 4.5 & 0.1 & 7.3 & - \\
\hline Broccoli & 37.0 & 89.9 & 3.3 & 0.2 & 5.5 & 2.6 \\
\hline Brussel's sprout & 45.0 & 85.2 & 4.9 & 0.4 & 8.3 & 1.5 \\
\hline Bengal gram leaves & 97.0 & 73.4 & 7.0 & 1.4 & 14.1 & - \\
\hline Cabbage & 24.0 & 92.4 & 1.3 & 0.2 & 5.4 & 1.5 \\
\hline Capsicum & 29.0 & 92.5 & 1.2 & 0.2 & 4.0 & 2.5 \\
\hline Chilli & 29.0 & 82.6 & 2.9 & 0.6 & 6.1 & 6.7 \\
\hline Carrot & 42.0 & 88.6 & 1.1 & 0.2 & 9.1 & 1.0 \\
\hline Coriander leaves & 44.0 & 66.3 & 3.3 & 0.6 & 6.3 & - \\
\hline Cassava & 157.0 & 59.4 & 0.7 & 0.2 & 38.1 & - \\
\hline Cauliflower & 27.0 & 91.0 & 2.7 & 0.2 & 5.2 & 0.9 \\
\hline Celery & 17.0 & 94.1 & 0.9 & 0.1 & 3.9 & 1.4 \\
\hline
\end{tabular}




\begin{tabular}{|l|c|c|c|c|c|c|}
\hline Cucumber & 18.0 & 96.3 & 0.4 & 0.1 & 2.5 & 0.6 \\
\hline Drumstick leaves & 92.0 & 76.0 & 6.7 & 1.7 & 12.7 & 0.9 \\
\hline Fenugreek leaves & 49.0 & 86.1 & 4.4 & 0.9 & 6.0 & 1.1 \\
\hline French bean & 30.0 & 62.0 & 6.3 & 0.1 & 29.8 & 4.0 \\
\hline Garlic & 32.0 & 90.1 & 1.9 & 0.2 & 7.1 & 0.8 \\
\hline Kale & 53.0 & 82.7 & 6.0 & 0.8 & 9.0 & 1.5 \\
\hline Knolkhol & 29.0 & 90.3 & 2.0 & 0.1 & 6.6 & 1.1 \\
\hline Leek & 77.0 & 78.9 & 1.8 & 0.1 & 17.2 & 1.3 \\
\hline Lettuce & 14.0 & 95.1 & 1.2 & 0.2 & 2.5 & 0.6 \\
\hline Mint & 48.0 & 84.9 & 4.8 & 0.6 & 5.8 & 2.0 \\
\hline Musk melon & 17.0 & 95.2 & 0.3 & 0.2 & 3.5 & 0.6 \\
\hline Mustard leaves & 34.0 & 89.8 & 4.0 & 0.6. & 3.2 & 1.5 \\
\hline Okra & 35.0 & 89.6 & 1.9 & 0.2 & 6.4 & 1.2 \\
\hline Onion & 50.0 & 86.6 & 1.2 & 0.1 & 11.1 & 0.5 \\
\hline Pea & 84.0 & 78.0 & 6.3 & 0.4 & 14.4 & 4.0 \\
\hline Potato & 97.0 & 74.7 & 1.6 & 0.1 & 22.6 & 1.6 \\
\hline Parsley & 16.0 & 90.0 & 2.2 & 0.3 & 1.3 & - \\
\hline Pointed gourd & 20.0 & 92.0 & 2.0 & 0.3 & 2.2 & 3.0 \\
\hline Pumpkin & 25.0 & 92.6 & 1.4 & 0.1 & 4.6 & 0.5 \\
\hline Radish & 17.0 & 94.4 & 0.7 & 0.1 & 3.4 & 0.7 \\
\hline Sponge gourd & 18.0 & 93.2 & 1.2 & 0.2 & 2.9 & 0.5 \\
\hline Spinach & 26.0 & 90.7 & 3.2 & 0.3 & 4.3 & - \\
\hline Spinach beet & 46.0 & 86.4 & 3.4 & 0.8 & 6.5 & 0.7 \\
\hline Sweet potato & 124.0 & 68.5 & 1.8 & 0.7 & 28.0 & 1.0 \\
\hline Tomato & 22.0 & 93.5 & 1.1 & 0.2 & 4.7 & 0.7 \\
\hline Turnip green & 67.0 & 8.9 & 4.0 & 1.5 & 9.4 & - \\
\hline Watermelon & 26.0 & 92.6 & 0.5 & 0.2 & 6.4 & 0.2 \\
\hline Nadroo (Lotus root) & 53.0 & 85.9 & 1.7 & 0.1 & 11.3 & - \\
\hline Yam & 102.0 & 74.0 & 1.5 & 0.2 & 24.0 & - \\
\hline Taro corm (Arvi) & 97.0 & 73.1 & 3.0 & 0.1 & 22.1 & - \\
\hline Giant Taro (Kachloo) & 71.0 & 81.2 & 0.6 & 0.1 & 17.0 & - \\
\hline Cowpea leaves & 51.0 & 84.6 & 4.3 & 0.2 & 8.0 & - \\
\hline & & & & & & \\
\hline
\end{tabular}

Table.2 Vitamin composition in vegetables per $100 \mathrm{~g}$ edible portion

\begin{tabular}{|l|c|c|c|c|c|}
\hline \multicolumn{1}{|c|}{ Vegetable crops } & $\begin{array}{c}\text { Vitamin A } \\
(\mathbf{I U})\end{array}$ & $\begin{array}{c}\text { Thiamin } \\
(\mathbf{m g})\end{array}$ & $\begin{array}{c}\text { Riboflavin } \\
(\mathbf{m g})\end{array}$ & $\begin{array}{c}\text { Niacin } \\
(\mathbf{m g})\end{array}$ & $\begin{array}{c}\text { Vitamin C } \\
(\mathbf{m g})\end{array}$ \\
\hline Amaranth & 9,108 & 0.03 & 0.30 & 1.2 & 99.9 \\
\hline Asparagus & 900 & 0.18 & 0.20 & 1.5 & 33.0 \\
\hline Basella & 12,276 & 0.03 & 0.16 & 0.5 & 87.0 \\
\hline Bittergourd & 208 & 0.07 & 0.09 & 0.5 & 88.0 \\
\hline Bottle gourd & traces & 0.03 & 0.01 & 0.2 & 6.0 \\
\hline Brinjal & 122 & 0.04 & 0.11 & 0.9 & 12.0 \\
\hline
\end{tabular}




\begin{tabular}{|c|c|c|c|c|c|}
\hline Broad bean & 15 & 0.08 & - & 0.8 & 12.0 \\
\hline Broccoli & 2,500 & 0.10 & 0.23 & 0.9 & 113.0 \\
\hline Brussel's sprout & 550 & 0.10 & 0.16 & 0.9 & 102 \\
\hline Cabbage & 130 & 0.05 & 0.05 & 0.03 & 47 \\
\hline Capsicum & 900 & 0.06 & 0.06 & 0.5 & 175 \\
\hline Chilli (green) & 454 & 0.06 & 0.03 & 0.6 & 111 \\
\hline Carrot & 11,000 & 0.06 & 0.05 & 0.6 & 8 \\
\hline Coriander leaves & 11,168 & 0.50 & 0.06 & - & 135 \\
\hline Cassava & 700 & 0.05 & 0.10 & 0.3 & 25 \\
\hline Cauliflower & 60 & 0.11 & 0.10 & 0.7 & 78 \\
\hline Celery & 240 & 0.03 & 0.03 & 0.3 & 9 \\
\hline Cucumber & 0.00 & 0.03 & 0.0 & 0.2 & 7 \\
\hline Drumstick leaves & 11,187 & 0.06 & 0.05 & 0.8 & 220 \\
\hline Fenugreek leaves & 3,861 & 0.04 & 0.31 & 0.8 & 52 \\
\hline French bean & 10 & 0.06 & 0.23 & 0.4 & 13 \\
\hline Garlic & 600 & 0.08 & 0.11 & 0.5 & 19 \\
\hline Kale & 10,000 & 0.16 & 0.26 & 2.1 & 186 \\
\hline Knolkhol & 20 & 0.06 & 0.04 & 0.3 & 66 \\
\hline Leek & 30 & 0.23 & 0.06 & 0.5 & 25 \\
\hline Lettuce & 900 & 0.06 & 0.06 & 0.3 & 8 \\
\hline Mint & 2,700 & 0.05 & 0.20 & 0.4 & 750 \\
\hline Musk melon & 279 & 0.11 & 0.08 & 0.3 & 26 \\
\hline Mustard leaves & 4,200 & 0.03 & - & - & 33 \\
\hline Okra & 172 & 0.07 & 0.10 & 0.6 & 13 \\
\hline Onion & 35 & 0.08 & 0.01 & 0.4 & 11 \\
\hline Pea & 640 & 0.35 & 0.14 & 2.4 & 27 \\
\hline Potato & 32 & 0.10 & 0.01 & 1.2 & 17 \\
\hline Parsley & 5,200 & 0.08 & 0.11 & 0.7 & 90 \\
\hline Pointed gourd & 252 & 0.05 & 0.06 & 0.5 & 29 \\
\hline Pumpkin & 2180 & 0.06 & 0.04 & 0.05 & 2 \\
\hline Radish & 5 & 0.06 & 0.04 & 0.05 & 15 \\
\hline Bengal gram leaves & 1564 & 0.09 & 0.10 & - & 61 \\
\hline Sponge gourd & 369 & 0.02 & 0.06 & 0.4 & 0 \\
\hline Spinach & 8100 & 0.10 & 0.20 & 0.6 & 51 \\
\hline Spinach beet & 5862 & 0.26 & 0.56 & 3.3 & 70 \\
\hline Sweet potato & 8800 & 0.10 & 0.06 & 0.6 & 21 \\
\hline Tomato & 900 & 0.06 & 0.04 & 0.7 & 23 \\
\hline Turnip green & 15691 & 0.31 & 0.57 & 5.4 & 180 \\
\hline Taro corm (Arvi) & 166 & 0.09 & 0.03 & - & 0 \\
\hline Giant Taro (Kachloo) & - & 0.31 & 0.57 & 5.4 & 180 \\
\hline Cowpea pods & 941 & 0.07 & 0.09 & 0.9 & 13 \\
\hline Yam & - & 0.1 & 0.01 & 0.8 & 15 \\
\hline
\end{tabular}


Table.3 Qualitative and quantitative carotenoid in vegetables

\begin{tabular}{|c|c|c|c|}
\hline $\begin{array}{l}\text { Vegetables- carotenoids } \\
\text { (mg/g fresh wt) }\end{array}$ & Total & $\beta$-carotene & Major carotenoids \\
\hline $\begin{array}{l}\text { Asparagus (Asparagus } \\
\text { officinalis L.) }\end{array}$ & 8.5 & $4.3-7.0$ & $\alpha$-carotene. Lutein, Violaxanthin, Neoxanthin \\
\hline $\begin{array}{l}\text { Bitter gourd (Momordica } \\
\text { charantia L.) }\end{array}$ & 5.3 & 2.3 & $\alpha$-carotene, $\beta$-carotene. Zeinoxanthin. Lutein \\
\hline $\begin{array}{l}\text { French bean (Phaseolus } \\
\text { vulgaris L.) }\end{array}$ & 17.1 & $2-4$ & $\begin{array}{l}\alpha \text {-carotene, } \beta \text {-carotene. Lutein 5,6- epoxide. } \\
\text { Neoxanthin, Violaxanthin }\end{array}$ \\
\hline $\begin{array}{l}\text { Broccoli (Brassica oleracea var. } \\
\text { italica Plenck.) }\end{array}$ & 42.4 & 4.8 & $\begin{array}{l}\text { â-carotene. Lutein, Isolutein, Luteoxanthin, } \\
\text { Violaxanthin, Neoxanthin, Chrysanthemaxanthin }\end{array}$ \\
\hline $\begin{array}{l}\text { Cabbage (Brassica oleracea } \\
\text { var. captita L.) }\end{array}$ & 8.9 & 0.8 & $\begin{array}{l}\beta \text {-carotene. Lutein, } \alpha \text {-carotene } 5,6 \text { - epoxide, } \\
\text { Neoxanthin, Violaxanthin, Chrysanthemaxanthin }\end{array}$ \\
\hline Carrots (Daucus carota L.) & $54-124$ & 76.0 & $\begin{array}{l}\beta \text {-carotene, } \beta \text {-carotene, J-carotene, â-Zeacarotene, } \\
\text { r-carotene, Neurosporene }\end{array}$ \\
\hline $\begin{array}{l}\text { Cauliflower (Brassica oleracea } \\
\text { var. captita L.) }\end{array}$ & 0.44 & 0.11 & $\beta$-carotene, Lutein, Violaxanthin, Neoxanthin \\
\hline Cucumber (Cucumis sativus L.) & 17.2 & 2.20 & $\alpha$-carotene, $\beta$-carotene and Cryptoxantin \\
\hline $\begin{array}{l}\text { Pepper (Green) (Capsicum } \\
\text { annuum L.) }\end{array}$ & 10.0 & 6.8 & Capsanthin, Capsorubin, Cryptocapsin, $\beta$-carotene \\
\hline Pepper (Red) & $127-284$ & $1.27-2.84$ & $\beta$-carotene, Violaxanthin, Neoxanthin \\
\hline Lettuce (Lactuca sativa $\mathrm{L}_{\text {.) }}$ & 68.0 & $10.8-24.5$ & $\beta$-carotene, Lutein, Violaxanthin, Neoxanthin \\
\hline Spinach (Spinacia oleraeea L. & 69.0 & 40.0 & $\begin{array}{l}\beta \text {-carotene. Lutein epoxide, Violaxanthin, Lutein, } \\
\text { Antheraxanthin, Neoxanthin }\end{array}$ \\
\hline $\begin{array}{l}\text { Tomato (Lycoopersicon } \\
\text { esculentum L.) }\end{array}$ & $70-190$ & 7.8 & Lycopene, â-carotene, Phytoene. \\
\hline
\end{tabular}

Source: Pigments in vegetables: Cholorophylls and Carotenoids, (Jeana Gross (1991). Van Nostrand Reinhold, New York

Table.4 Groups of coloured vegetable crops

\begin{tabular}{|l|l|}
\hline $\begin{array}{l}\text { Colours } \\
\text { vegetables }\end{array}$ & \multicolumn{1}{c|}{ Groups of coloured vegetable crops } \\
\hline Green & $\begin{array}{l}\text { Artichoke, asparagus, amaranth, broccoli, Brussels sprout, celery, squash, Chinese } \\
\text { cabbage, cucumber, endive, egg plant, beans green, cabbage green, onion green, } \\
\text { pepper green, leek, lettuce, okra, peas, spinach, snap pea, watercress. }\end{array}$ \\
\hline White & $\begin{array}{l}\text { Cauliflower, garlic, Jerusalem artichoke, kholrabi, egg plant, onion, parsnip, } \\
\text { potato, shallot, turnip, white corn, white radish. }\end{array}$ \\
\hline Red & $\begin{array}{l}\text { Beet, radish, red capsicum, red pepper, red onion, red potato, tomato, watermelon, } \\
\text { red amaranth }\end{array}$ \\
\hline Yellow/Orange & $\begin{array}{l}\text { Pumpkin and squashes, carrot, sweet corn, sweet potato, beet yellow, yellow } \\
\text { capsicum, potato yellow, yellow tomato, watermelon yellow. }\end{array}$ \\
\hline Blue/Purple & $\begin{array}{l}\text { Egg plant, purple potato, purple cabbage, black carrot, purple cowpea, purple } \\
\text { dolichos bean, purple capsicum and chilli }\end{array}$ \\
\hline
\end{tabular}


Table.5 Vegetable production and consumption

\begin{tabular}{|c|c|c|c|c|c|c|c|c|c|c|c|c|c|c|c|c|c|}
\hline \multirow[t]{2}{*}{$\begin{array}{l}\text { S. } \\
\text { No. }\end{array}$} & \multirow{2}{*}{$\begin{array}{l}\text { Name of } \\
\text { Fruits and } \\
\text { vegetables }\end{array}$} & \multicolumn{2}{|c|}{$\begin{array}{c}\text { Production } \\
\text { (kg) }\end{array}$} & \multicolumn{2}{|c|}{$\begin{array}{l}\text { Consumption } \\
\text { (gm /day) }\end{array}$} & \multicolumn{2}{|c|}{ Protein (gm) } & \multicolumn{2}{|c|}{$\begin{array}{l}\text { Energy } \\
\text { (Kcal) }\end{array}$} & \multicolumn{2}{|c|}{ Calcium (mg) } & \multicolumn{2}{|c|}{ Iron (mg) } & \multicolumn{2}{|c|}{$\begin{array}{l}\text { Vitamin A } \\
\text { (Carotene) } \mu \mathrm{g}\end{array}$} & \multicolumn{2}{|c|}{$\begin{array}{c}\text { Vitamin C } \\
\text { (mg) }\end{array}$} \\
\hline & & $\mathrm{BF}$ & $\mathrm{AF}$ & $\mathrm{BF}$ & $\mathrm{AF}$ & $\mathrm{BF}$ & $\mathrm{AF}$ & $\mathrm{BF}$ & $\mathrm{AF}$ & $\mathrm{BF}$ & $\mathrm{AF}$ & $\mathrm{BF}$ & $\mathrm{AF}$ & $\mathrm{BF}$ & $\mathrm{AF}$ & $\mathrm{BF}$ & $\mathrm{AF}$ \\
\hline 1. & Spinach & 0.0 & 25.0 & 50.0 & 70.0 & 1.0 & 1.4 & 13.0 & 18.2 & 36.5 & 51.1 & 0.57 & 0.8 & 2790 & 3906 & 14.0 & 19.6 \\
\hline 2. & Amranth & 10.0 & 28.0 & 40.0 & 65.0 & 2.08 & 3.38 & 15.2 & 24.7 & 132 & 214.5 & 7.48 & 12.15 & 0.0 & 0.0 & 71.6 & 116.35 \\
\hline 3. & Radish leaves & 25.0 & 45.0 & 60.0 & 70.0 & 2.28 & 2.66 & 16.8 & 19.6 & 159 & 185.5 & 0.54 & 0.63 & 3177 & 3706 & 48.6 & 56.7 \\
\hline 4. & $\begin{array}{c}\text { Safflower } \\
\text { leaves }\end{array}$ & 0.0 & 48.0 & 0.0 & 65.0 & 0.0 & 1.6 & 0.0 & 21.4 & 0.0 & 120.2 & 0.0 & 3.7 & 0.0 & 2301 & 0.0 & 9.75 \\
\hline 5. & Fenugreek & 0.0 & 28.0 & 25.0 & 45.0 & 0.0 & 2.0 & 0.0 & 22.0 & 0.0 & 177.7 & 0.0 & 0.86 & 0.0 & 1053 & 0.0 & 23.4 \\
\hline 6. & $\begin{array}{c}\text { Bengal gram } \\
\text { leaves }\end{array}$ & 20.0 & 35.0 & 45.0 & 55.0 & 3.1 & 3.85 & 43.6 & 53.3 & 153 & 187 & 10.7 & 13.0 & 0.0 & 0.0 & 0.0 & 0.0 \\
\hline 7. & Coriander & 2.0 & 3.0 & 5.0 & 10.0 & 0.16 & 0.33 & 2.0 & 4.4 & 9.2 & 18.4 & 0.07 & 0.14 & 346 & 692 & 6.75 & 13.5 \\
\hline 8. & Bottle Gourd & 12.0 & 26.0 & 60.0 & 75.0 & 0.12 & 0.15 & 7.2 & 9.0 & 12.0 & 15.0 & 0.276 & 0.34 & 0.0 & 0.0 & 0.0 & 0.0 \\
\hline 9. & Pumpkin & 12.0 & 35.0 & 70.0 & 75.0 & 0.98 & 1.05 & 17.5 & 18.7 & 7.0 & 7.5 & 0.308 & 0.33 & 35.0 & 37.5 & 1.4 & 1.5 \\
\hline 10. & Bitter Gourd & 15.0 & 38.0 & 25.0 & 35.0 & 0.4 & 0.56 & 6.25 & 8.75 & 5.0 & 7.0 & 0.15 & 0.21 & 31.5 & 44.1 & 22.0 & 30.8 \\
\hline 11. & Brinjal & 22.0 & 45.0 & 40.0 & 60.0 & 0.56 & 0.84 & 9.6 & 14.4 & 7.2 & 10.8 & 0.15 & 0.48 & 29.6 & 44.4 & 4.8 & 7.2 \\
\hline 12. & Lady finger & 10.0 & 45.0 & 35.0 & 55.0 & 0.66 & 1.0 & 12.5 & 19.2 & 23.1 & 36.3 & 0.12 & 0.19 & 18.2 & 28.6 & 4.55 & 7.15 \\
\hline 13. & Radish & 22.0 & 55.0 & 30.0 & 55.0 & 0.21 & 0.68 & 5.1 & 9.35 & 10.5 & 19.25 & 0.12 & 0.22 & 0.9 & 1.65 & 4.5 & 8.25 \\
\hline & Average & - & - & 37.30 & 56.5 & 0.88 & 1.5 & 11.4 & 18.69 & 42.6 & 80.78 & 1.57 & 2.54 & 494.4 & 908.7 & 13.7 & 22.63 \\
\hline
\end{tabular}

$\mathrm{BF}=$ Before intervention. $\mathrm{AF}=$ After intervention 
Fig.1 Nutritional garden model established
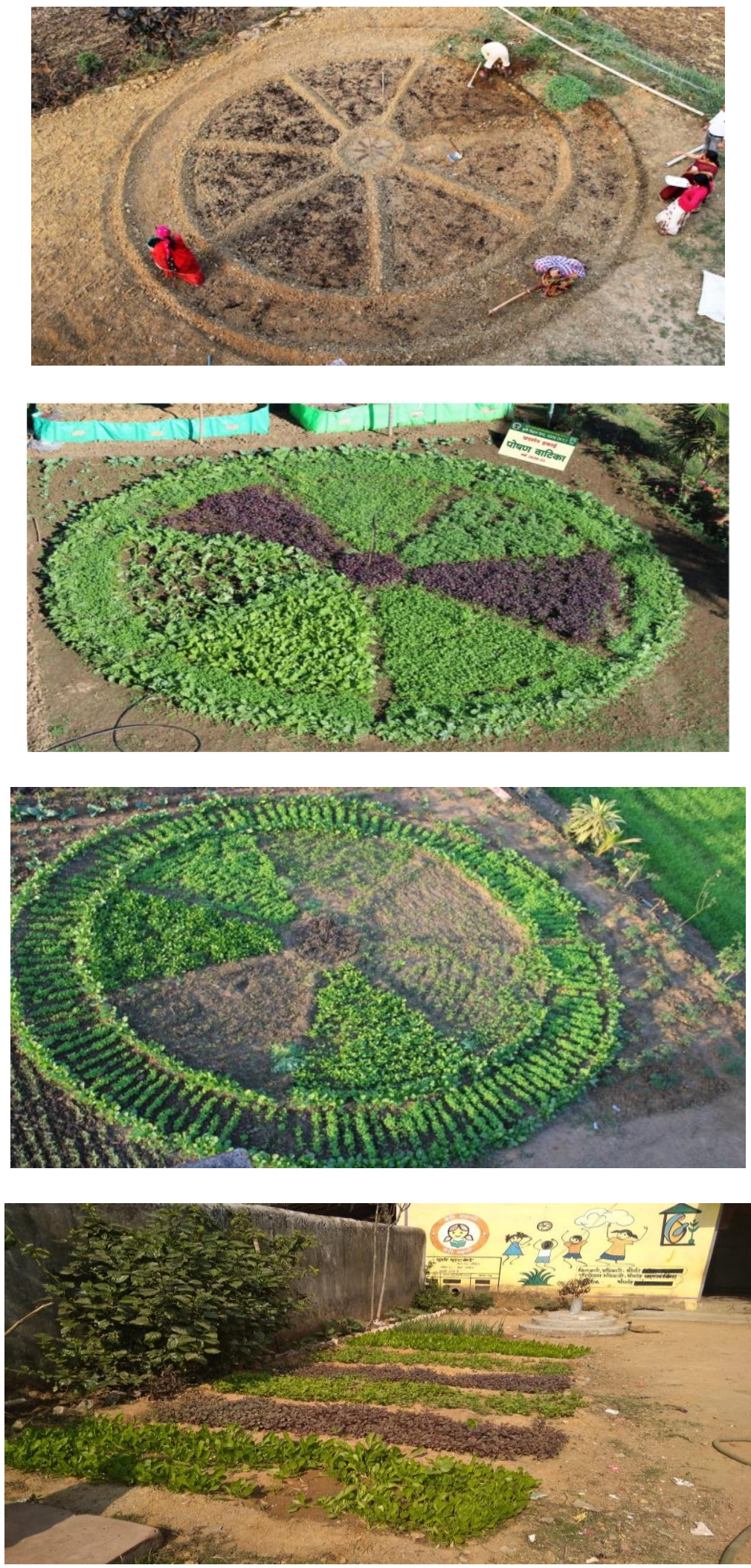

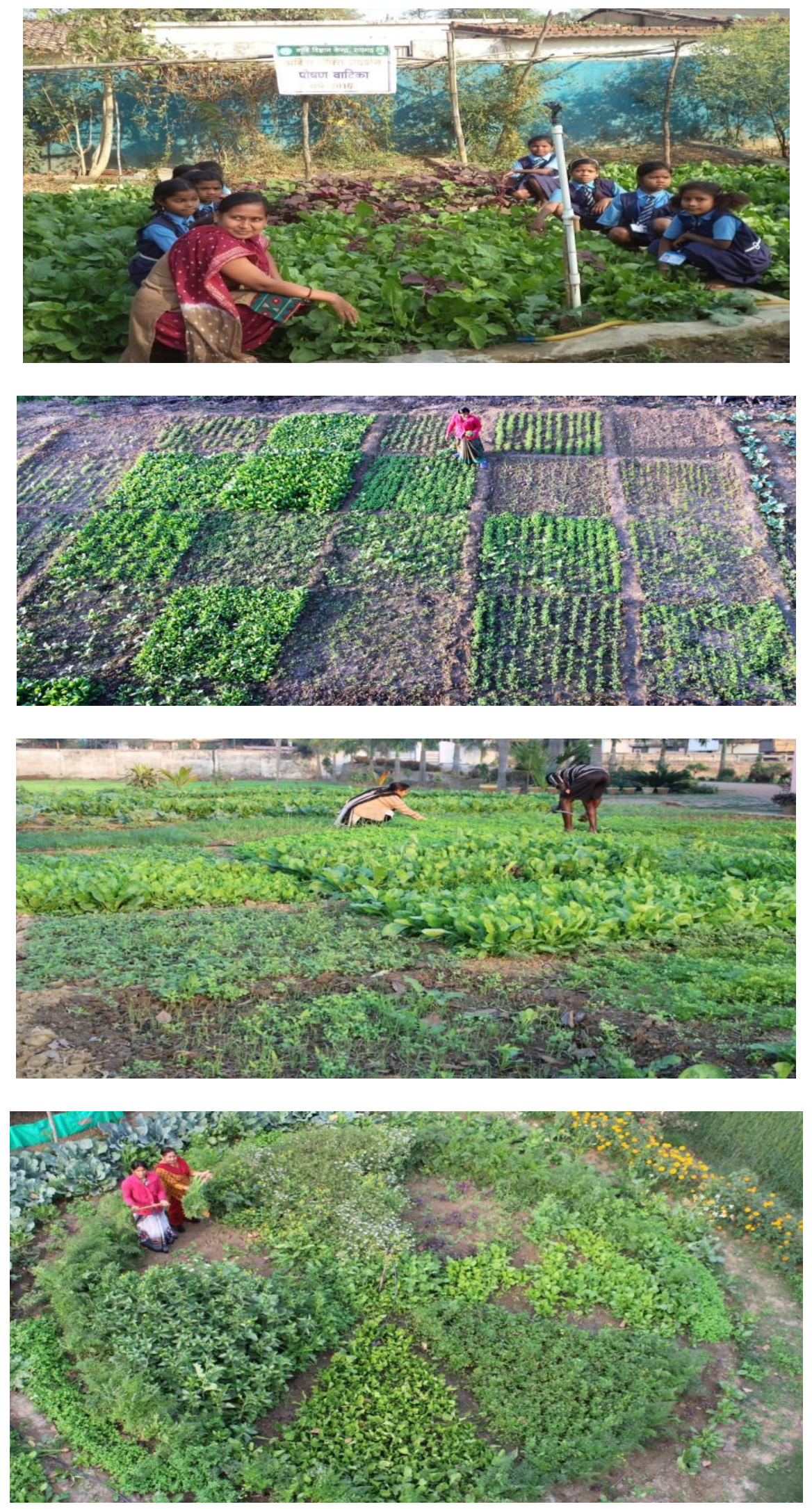
Vegetables help combat malnutrition and diversify diets. Dietary diversification balances the diet by enhancing the supply of essential micro-nutrients leading to improved health, such as improving functions of the whole body, disease prevention, and delayed disease progression. The dietary diversity was evident clearly which lead to reduction in malnutrition.

In conclusion the present study was carried out on nutritional garden towards improving nutritional security through production of vegetables and fruits, their consumption are meager especially among the farm households. Now-a-days people are more health conscious and good food shall be our medicine. Increased consumption of fruits and vegetables is one of the easiest and cheapest ways of enhancing health. Many underutilized fruits and vegetables, which are rich sources of photochemicals are to be cultivated in backyard nutritional gardens. There is an increasing demand for indigenous, location specific underutilized vegetables and fruits. Homestead nutritional gardening is a low cost sustainable approach for mitigating malnutrition especially for farm households. Home gardening contributes to household food security by providing direct access to food that can be harvested, prepared and fed to family members, often on a daily basis. Nutritional gardening may be done with virtually no economic resources, using locally available planting materials, green manures, "live" fencing and indigenous methods of plant protection. Thus, home gardening is a production system that fits well in family farming system. It is concluded that establishment of nutritional gardens had immense role in tackling the problem of malnutrition and micronutrients deficiencies in farm households.

Conflict of interest: Authors have declared that no conflict/ competing interests exist.

\section{References}

Awasthi, N., Sahu, A., Chandrakala and Singh, K. (2016). Advances in Social Res., 2(1), 49-51

Chayal, K., Dhaka, B. L., Poonia, M. K. and Bairwa, R. K. (2013). Asian J Home Sci., 8(2): 607-09.

Christanty, L. (1990). Home gardens in tropical Asia, with special reference to Indonesia, In: Landauer K., and Brazil M., ed. Tropical home gardens, 9-20, Tokyo, Japan: United Nations University Press.

Dauchet, L., Amouyel, P., Hercberg, S. and Dallongeville, J. (2006). Fruit and vegetable consumption and risk of coronary heart disease: a metaanalysis of cohort studies. J. Nutr., 136(10): 2588-93.

Dias, J. S. and Ortiz, R. (2013). New srategies and approaches for improving vegetable cultivars. In: Nath, P.(ed.) The basics of Human civilization- Food, Agriculture and Humanity, Present scenario, Dr. P. N. Agri. Sci. Foundation(PNASF). Bangalore and New India Publishing Agency (NIPA), New Delhi, pp 349381.

FAO (2009). Food and Agriculture Organisation of the United Nations, Rome, Italy. http:// www.fao.org

FAO (2011). Food and Agriculture Organisation of the United Nations, Rome, Italy. http:// www.fao.org

Goraya, N., Simoni, J., Jo, C.H. and Wesson, D.E. (2013). A comparison of treating metabolic acidosis in CKD stage 4 hypertensive kidney disease with fruits and vegetables or sodium bicarbonate, Clin. J. Am. Soc. Nephrol., 8: 371-81.

Hall, J. N., Moore, S., Harper, S. B. and Lynch, J. W. (2009). Global variability in fruit and vegetable consumption. Am. J. Prev. Med., 36: 402-409

Howarth, N.C., Saltzman, E. and Roberts, 
S.B. (2001). Dietary fiber and weight regulation. Nutr, Rev., 59(5): 129-139.

Hartley, L., Igbinedion, E., Holmes J. et al., (2013). Increased consumption of fruit and vegetables for the primary prevention of cardiovascular diseases, Cochrane Database Syst. Review., 6: CD009874.

He, F..J., Nowson, C.A., Lucas, M. and MacGregor, G.A. (2007). Increased consumption of fruit and vegetables is related to a reduced risk of coronary heart disease: meta-analysis of cohort studies. J. Hum. Hypertens, 21(9): 71728.

Indumathi, K., Shanmugam, P.S. and Tamilselvan, N. (2012). Nutrition garden as a valuable intervention to fight malnutrition in rural India. Proceeding of Global Conference on Horticulture for food, nutrition and livelihood options, Bhubaneswar, Odisha, India 28-31 May, p.19.

Jana, H. (2015) Rashtriya Krishi, 10(2),13-16. Jindal, S. K. and Dhaliwal, M. S. (2017). Development of vegetable nutrition garden model for diet diversification and improved nutrition security of urban and peri-urban households. International Journal of Horticulture., 7 (24): 219-28.

Johnson,W. C., Alemu, B., Msaki, T. P. et al., (2000). Improving household food security: institutions, gender and integrated approaches, Davis CA, USA: Paper prepared for the broadening access and strengthening input market systems (BASIS) collaborative research support project (CRSP).

Kanungsukkasem, U., Ng, N., Minh, H.V. et al., (2009). Fruit and vegetable consumption in rural adults population in INDEPTH HDSS sites in Asia. Glob Health Action. 2: 35-43.

Leenders, M., Sluijs, I., Ros, M. M. et al., (2013). Fruit and vegetable consumption and mortality: European prospective investigation into cancer and nutrition. Am. J. Epidemiol., 178: 590-602.

Lim, S. S., Vos, T., Flaxman, A. D. et al., (2012). A comparative risk assessment of burden of disease and injury attributable to 67 risk factors and risk factor clusters in 21 regions, 1990-2010: a systematic analysis for the Global Burden of Disease Study 2010. Lancet., 380: 2224-60.

Marsh, R. (1998). Building on traditional gardening to improve household food security. Food Nutr. Agric., 22: 4-14.

Macready, A.L., George, T.W., Chong, M.F. et al., (2014). Flavonoid-rich fruit and vegetables improve microvascular reactivity and inflammatory status in men at risk of cardiovascular disease FLAVURS: a randomized controlled trial. Am. J. Clin. Nutr., 99: 479-89.

Melina (2012). Realistic and livable tips for a healthy lifestyle. Accessed April 19, 2012. Mitchell, R. and Hanstad, T. (2004). Small home garden plots and sustainable livelihoods for the poor, Rome. Italy: LSP Working Paper 11.

NCCDPHP (2013). National Center for Chronic Disease Prevention and Health Promotion, State Indicator Report on Fruits and Vegetables, Atlanta, GA: Centers for Disease Control and Prevention, US Dept of Health and Human Services.

NIN (2011). Dietary Guidelines for Indians A Manual, National Institute of Nutrition, Hyderabad, India,p.127.

Nyaradi, A., Foster, J.K., Hickling, S. et al., (2014). Prospective associations between dietary patterns and cognitive performance during adolescence. $J$. Child Psychol. Psychiatry., 55: 101724.

Satheannoppakao, W., Aekplakorn, W. and Pradipasen, M. (2009). Fruit and 
vegetable consumption and its recommended intake associated with sociodemographic factors:

Thailand National Health Examination Survey III. Public Health Nutr., 12: 2192-98. Sharma, J. P. (2009). Principles of Vegetable Breeding. Kalyani Publishers, New Delhi.p.288312.

Shackleton, C.M., Paumgarten, F. and Cocks, M. L. (2008). Household attributes promote diversity of tree holdings in rural areas, South Africa. Agrofor. Syst., 72: $221-30$.

Singh, H.P. (2012). Trend of horticultural research particularly vegetables in India and its regional prospects. Proc. Proc. Regional Symposium on high value vegetables in South-east Asia: Production, Supply and Demand (SEAVEG 2012). Chiang Mai, Thailand 24-26 January p.34.

Singh, V., Yadav, K. S. and Tripathi, A. K. (2018.). Kitchen gardening: A promising approach towards improving nutritional security in rural households. International Journal of Microbiology Research., 10 (5): 1216-19.
Somsri, P., Satheannoppakao, W., Tipayamongkholgul, M., Vatanasomboon, P., and Kasemsup, R. (2016). A cosmetic content-based nutrition education program improves fruit and vegetable consumption among grade 11 Thai students. J. Nutr. Educ. Behav., 1-9.

Talukder, A., Kiess, L., Huq, N., De-pee, S., Darton-Hill and Bloem, M.W. (2002). Increasing the production and consumption of vitamin A-rich fruits and vegetables: Lesson learned in taking the Bangladesh homestead gardening programme to national scale. Food Nutr. Bull., 21 (2): 165-172.

Tohill, B.C. (2005). Dietary intake of fruit and vegetables and management of body weight. World Health Organization (WHO). 1-52.

Vani Bhushanam, G. and Usha Rani, M. (2013). Am. Int. J. Res. in Formal, Applied and Natural Sci., 3(1): 78-81.

Zhong, X., Fang, Y. J., Pan, Z.Z. et al., (2014). Dietary fiber and fiber fraction intakes and colorectal cancer risk in Chinese adults. Nutr. Cancer., 66: 351361

\section{How to cite this article:}

Manisha Choudhary, S. P. Singh and Chanchala Rani Patel. 2021. Nutritional Garden: A Nutrition Sensitive Promising Approach towards Improving Nutritional Security in Farm Households. Int.J.Curr.Microbiol.App.Sci. 10(02): 981-994. doi: https://doi.org/10.20546/ijcmas.2021.1002.116 